\title{
Calidad del servicio municipal desde la perspectiva del ciudadano
}

\author{
Regner Nicolás Castillo Salazar \\ rcastillos@ucv.edu.pe \\ Universidad César Vallejo \\ Maridza Cárdenas Murrieta \\ ccardenasmur@ucvvirtual.edu.pe \\ Universidad César Vallejo \\ Gabriela del Pilar Palomino Alvarado \\ gabypal11@hotmail.com \\ Universidad César Vallejo
}

\section{RESUMEN}

El propósito de la investigación fue, caracterizar la calidad del servicio municipal desde la perspectiva del ciudadano, la metodología empleada fue de tipo básica, diseño no experimental, descriptiva, con una muestra de 157 ciudadanos, la técnica aplicada fue la encuesta y el cuestionario como instrumento. Los resultados muestran las características de la calidad del servicio municipal, desde la perspectiva del ciudadano a través del Modelo SERVQUAL, identificándose que; el 42,2\% de los ciudadanos se encuentran AED y TED con los elementos tangibles, por otro lado, respecto a la fiabilidad el $53.1 \%$, capacidad de respuesta el $45.5 \%$, seguridad el $52.7 \%$ y empatía el $48.1 \%$ mencionan que están TDA y ADA. Los nudos críticos de gestión que dificultan la calidad de los servicios, son: las deficiencias en el seguimiento del servicio, la compresión al usuario, las dificultades para resolver reclamos, la atención virtual deficiente y las instalaciones no modernas. Se recomienda realizar una propuesta innovadora en la gestión de la calidad del servicio municipal, para la solución a esta problemática.

Palabras claves: Calidad de servicio; municipal; modelo SERVQUAL; nudos críticos; ciudadano; elementos tangibles. 


\title{
Quality of municipal service from the perspective of the citizen
}

\begin{abstract}
The purpose of the research was to characterize the quality of the municipal service from the citizen perspective, the methodology used was basic type, non-experimental descriptive design, with a sample of 157 citizens, the applied technique was the survey and the questionnaire as an instrument. The results show the characteristics of the quality of the municipal service, from the perspective of the citizen through the SERVQUAL Model, identifying that; $42.2 \%$ of citizens are AED and TED with tangible elements, on the other hand, in terms of reliability $53.1 \%$, response capacity $45.5 \%$, security $52.7 \%$ and empathy $48.1 \%$ mention that they are ADD and ADA. The critical management nodes that hinder the quality of services are: deficiencies in the monitoring of the service, user compression, difficulties in resolving complaints, deficient virtual attention and lack of modern facilities. It is recommended to make an innovative proposal in the management of the quality of the municipal service, to solve this problem.
\end{abstract}

Keywords: Quality of service; municipal; SERVQUAL model; critical knots; citizen; tangible items. 


\section{Qualidade do serviço municipal na ótica do cidadão}

\section{RESUMO}

O objetivo da pesquisa foi caracterizar a qualidade do serviço municipal na perspectiva do cidadão, a metodologia utilizada foi de tipo básico, não experimental, descritiva, com uma amostra de 157 cidadãos, a técnica aplicada foi o inquérito e o questionário como instrumento. Os resultados mostram as características da qualidade do serviço municipal, na perspectiva do cidadão por meio do Modelo SERVQUAL, identificando que; $42,2 \%$ dos cidadãos são AED e TED com elementos tangíveis, por outro lado, quanto à confiabilidade $53,1 \%$, capacidade de resposta $45,5 \%$, segurança $52,7 \%$ e empatia $48,1 \%$ mencionam que são ADD e ADA. Os nós críticos de gestão que dificultam a qualidade dos serviços são: deficiências no monitoramento do serviço, compressão do usuário, dificuldade de resolução de reclamações, atendimento virtual deficiente e instalações descomplicadas. Recomenda-se fazer uma proposta inovadora na gestão da qualidade do serviço municipal, para a solução deste problema.

Palavras-chave: Qualidade de serviço; municipal; Modelo SERVQUAL; nós críticos; cidadão; Itens tangíveis.

Artículo recibido: 03 nov. 2020 Aceptado para publicación: 07 dic. 2020 Correspondencia: rcastillos@ucv.edu.pe. Conflictos de Interés: Ninguna que declarar 


\section{INTRODUCCIÓN}

La calidad de los servicios se genera con la satisfacción de las necesidades y expectativas del cliente o ciudadano, el estado lo realiza a través de diferentes organismos y en sus normas vigentes. No obstante, la gestión de los gobiernos municipales en los diferentes países es deficiente para brindar calidad. La innovación se está convirtiendo en estos finales de tiempo en un aliciente de cambio para muchas instituciones públicas de los países más representativos de Europa o América del Norte, poniendo por delante el desarrollo de gobiernos basados en la transformación desde los locales, centrales, hasta los nacionales tomando como punto de partida la sistematización de los procesos con el uso de la tecnología (Eriksson et al., 2017)

En América Latina, existen experiencias similares menos conocidas en Chile, México y Argentina que impulsaron una gestión innovadora (Mauro et al., 2019), siendo la primera, el de menor resultado de satisfacción a los usuarios, por no tener las herramientas para optimizar su gestión (Zhao et al., 2020). Las deficiencias se dan en la mayoría de municipalidades del sur de América Latina, pero la innovación permitirá cubrir esa brecha. Según el Instituto Nacional de Estadística e Informática (INEI, 2018), en el Perú existen 1643 municipalidades, que por su propia naturaleza, están enfocadas en la gestión directa y autónoma de los recursos que permitan implementar nuevas políticas y servicios en el sector de vivienda, turismo, salud, ambiente, por su mayor acercamiento con la población; sin embargo, existen deficiencias sobre todo en los lugares más alejados con menos recursos presupuestario y de personal con poca educación en gestión pública, generando insatisfacción por parte de los pobladores.

Si bien es cierto, contamos con una política de modernización del estado que permite desarrollar servicios para satisfacer las necesidades, requerimientos y/o expectativas de los ciudadanos, estás aún no se han implementado en un 100\%. Tal es así, que en el DS 004-2013-PCM, establece que las entidades públicas deben regirse bajo la estrategia de gobierno abierto, electrónico e interarticulación institucional, para garantizar que la ejecución presupuestal sea eficiente y responda a estas demandas. Para ello exige que las entidades públicas inicien el proceso de cambio de una gestión funcional o vertical a una gestión por procesos u horizontal para resultados, de acuerdo a estándares internacionales de calidad basado en la norma ISO 9001:2015. Esta política propuesta por la Presidencia del Consejo de Ministros, está articulada al Centro de Planeamiento Estratégico (CEPLAN) que garantiza que la visión de la organización esté alineada a la visión del 
gobierno regional, del país y del mundo, cuyo objetivo es generar calidad en el sistema educativo, salud, seguridad, es decir, desarrollo sostenible en la región San Martín (Durand Guevara, 2014).

\section{Alcances de calidad en los servicios municipales desde la perspectiva del ciudadano.}

La evaluación mediante el modelo SERVQUAL según, Živković et al., (2019) nos menciona que, la calidad del servicio municipal, desde el punto de vista de los clientes, no es satisfactoria, es decir, existen brechas significativas entre las expectativas de los clientes y su percepción de cada determinante de la calidad del servicio, especialmente la confiabilidad y responsabilidad. de servicio. Asimismo, Emrah et al., (2019) indican que la mayor diferencia entre percepción y expectativa se encuentra en el factor de confiabilidad con $28,92 \%$. El factor tangible ha tenido el porcentaje de menor importancia con $12,42 \%$.

Del mismo modo, Alam \& Mondal, (2019) el servicio de saneamiento cumple con un nivel de satisfacción moderado $(58,5 \%)$ de expectativa pública en el barrio pobre ferroviario de la ciudad de Khulna. Esta metodología combinada proporciona una idea clara de la calidad del servicio de saneamiento de una manera más simple sin ningún requisito de datos cuantitativos que será útil en la planificación y gestión adecuadas del servicio municipal. Del mismo modo, Kansara, (2020), menciona que los ciudadanos no tienen confianza en los servicios municipales y los bienes tangibles son deficientes para el buen desempeño de los trabajadores.

Desde otra perspectiva, Bostanc1 \& Erdem, (2020) mencionan que, la calidad de los servicios deben ser percibidos a través de la satisfacción del ciudadano, las cuales aportará en el futuro la búsqueda de soluciones para incrementar la calidad de vida y los niveles de satisfacción. Del mismo modo, Dris Kharroub \& Cothman Mansour, (2019), refiere que la planificación estratégica tiene una correlación e impacto positivo en la calidad del servicio brindado a los ciudadanos. Por otro lado Moletsane et al., (2014) presenta hallazgo que indican percepciones negativas sobre la calidad de los servicios prestados por el municipio.

Para medir la calidad del servicio se encuentra el aporte de Parasuraman et al., (1985) indica que, son las capacidades que tienen las instituciones para generar valor de intercambio para el ciudadano a través de los servicios que desarrollan a través de los 
elementos tangibles, generando fiabilidad, con capacidad de respuesta, dotando de seguridad, con un trato de empatía.

Por otro lado, Mirabal et al., (2018), la realización del diagnóstico en el municipio los Palacios permitió constatar que predomina una visión sectorial de la calidad de vida por lo que se adolece de un proceso de gestión pública de la calidad de vida con enfoque integral desde los gobiernos locales y la Administración Pública. Asimismo, PastorSeller, (2015), los gobiernos locales se deben integrar a las necesidades de la población de tal manera que permita la elaboración de servicios acordes con los cambios y procesos de crecimiento de su entorno. Octavio \& Cruz, (2016), el colaborador de la municipalidad que tiene altos conocimientos, buena actitud y aptitud, genera condiciones y aporta a mejorar la calidad de los servicios públicos por ello la importancia de fortalecer las competencias de los servidores municipales. Más del 50\% de las dimensiones e indicadores evaluados son relacionados a la capacidad que tenga el personal de la entidad a través de sus competencias y aptitudes para el logro de los resultados.

La calidad de los servicios se ve enfrascada en el proceso que realizan las entidades, con la finalidad de brindar el mejor servicio de atención a los usuarios el cual es uno de los objetivos institucionales, es decir, centrado en las necesidades del usuario; así mismo, se debe generar desde la parte interna de las instituciones públicas, es decir, cada miembro debe estar en la capacidad de cambiar los antiguos paradigmas y volcarse al uso de nuevos sistemas de gestión de calidad (Idrovo-Toala et al., 2020).

Para mejorar la calidad de los servicios de una institución tenemos que tener definidos los conceptos y los procesos por los cuales se ha pasado a través de los años, generando cambios y mejoras importantes para lograr un mejor desempeño de las organizaciones, se establece también las filosofías y la evolución de las mismas desde las perspectivas de los funcionarios y además de las perspectivas del usuario o consumidor de los servicios, es subjetivo poder determinarlo. Según, Gobena, (2019), el trabajador municipal es quien brinda y abandera la calidad de servicios, porque es quien brinda las condiciones para que el ciudadano obtenga un experiencia satisfactoria, permitiendo su acceso continuo.

Asimismo, Emrah et al., (2019), indica son los ciudadano quienes deben hacer la evaluación de los servicios, para lograr conocer las condiciones en las cuales favorece al ciudadano, porque es el quien valora a través de su percepción logrando satisfacer las expectativas. Del mismo modo, Benito et al., (2019), indica que con el uso de políticas 
unidas y convergentes se puede mejorar la calidad de los servicios en las instituciones públicas, logrando bienestar en favor del ciudadano.

Por otro lado, Psomas et al., (2017), la satisfacción laboral, brinda una mejor calidad en los servicios, las autoridades deben sumar en el desarrollo de los mismo para que así los ciudadanos se sientan más satisfechos, por ello la importancia de tener trabajadores con óptimas condiciones. También, menciona que la calidad del servicio se encuentra basado en las habilidades que tiene el personal de las municipalidades para brindarlo, por lo cual tienen que estar constantemente capacitados, también el desarrollo de estrategias mediante el uso de incentivos permite el incremento del nivel de calidad de los servicios en las municipalidades.

De otro lado, Lo Storto, (2016) la calidad del servicio que brindan en los centros de atención al cliente de Sizakala dentro del Municipio Ethekwini de Sudáfrica, tienen que mejorar su dimensión de capacidad de respuesta y la importancia de tener como prioridad el fácil acceso de los servicios. De igual manera, Mhlongo et al., (2016), menciona que, los factores que determinan el modelo de gestión de calidad en las municipalidades e instituciones de Rajabhat, Tailandia, para que sean de éxito requieren de autoridades que sean líderes, realicen un adecuado entrenamiento; se complementen con una adecuada estructura organizacional, desarrollo de la comunicación interna y externa, generar incentivos, realizar de forma continua las mediciones y evaluación y generar el trabajo en equipo.

Finalmente según Asubonteng et al., (1996) menciona que la manera de medir más frecuente y popularizada de la calidad del servicio es el Modelo SERVQUAL, un instrumento desarrollado por Parasuraman et al., (1985; 1988). La misma que no solo ha sido objeto de citaciones en importantes artículos relacionadas al marketing en empresas privadas, además también es utilizada para la medición de los servicios de instituciones públicas.

\section{ESTRATEGIAS METODOLÓGICAS O MATERIALES Y MÉTODOS}

El tipo de investigación fue básica, de diseño no experimental, cuantitativo, descriptivo tuvo como propósito caracterizar la calidad del servicio municipal desde la perspectiva del ciudadano, en el distrito de Morales, además también se propuso, identificar los nudos críticos de gestión que dificultan la calidad de los servicios en el municipio. Se aplicó 
como técnica la encuesta y como instrumento el cuestionario Modelo SERVQUAL, a 157 ciudadanos. Se analizó los datos mediante el Software SPSS 25.

\section{RESULTADOS Y DISCUSIÓN}

Los datos descriptivos de la investigación, se desarrollaron a través un cuestionario de la variable Calidad de servicio, se describe en la siguiente tabla:

Tabla 1. Datos descriptivos de los ciudadanos usuarios del Municipio

\begin{tabular}{llcr}
\hline & Indicadores & fi & \% \\
\hline \multirow{3}{*}{ Edad } & 18 a 25 años & $\mathbf{5 1}$ & $\mathbf{3 2 . 5}$ \\
& 26 a 33 años & 40 & 25.5 \\
& 34 a 41 años & 37 & 23.6 \\
& 42 a 49 años & 14 & 8.9 \\
& 50 años a mas & 15 & 9.6 \\
& Primaria & 9 & 5.7 \\
Grado de & Secundaria & 18 & 11.5 \\
& Técnico incompleto & 10 & 6.4 \\
& Técnico completo & 16 & 10.2 \\
Genero & Superior Univ. Incompleto & $\mathbf{4 0}$ & $\mathbf{2 5 . 5}$ \\
& Superior Univ. completo & 38 & 24.2 \\
& Maestría & 23 & 14.6 \\
& Doctorado & 3 & 1.9 \\
& Masculino & 64 & 40.8 \\
& Femenino & $\mathbf{9 3}$ & $\mathbf{5 9 . 2}$ \\
& Soltero (a) & $\mathbf{9 0}$ & $\mathbf{5 7 . 3}$ \\
& Casado (a) & 35 & 22.3 \\
& Conviviente & 24 & 15.3 \\
& Viudo (a) & 0 & 0.0 \\
& Divorciado & 8 & 5.1 \\
& Total & $\mathbf{1 5 7}$ & $\mathbf{1 0 0 . 0}$ \\
\hline
\end{tabular}

Fuente: Encuesta a los ciudadanos del distrito de Morales

Interpretación: De los 157 ciudadanos encuestados, el 32.5\% (51), son entre las edades de 18 a 25 años, el $25.5 \%$ (40) cuentan con educación universitaria incompleta, el 59.2\% (93), es del género femenino y el 57.3\% (90), se encuentran en estado civil soltero.

Caracterizar la calidad de los servicios en el Municipio del Distrito de Morales.

Tabla 2. Calidad de servicio, desde la percepción del ciudadano

\begin{tabular}{ccccccc}
\hline $\begin{array}{c}\text { Dimensi } \\
\text { ón }\end{array}$ & Indicadores & TDA & ADA & NDANED & AED & TED \\
\hline $\begin{array}{c}\text { Element } \\
\text { os }\end{array}$ & $\begin{array}{l}\text { Equipos tecnológicos } \\
\text { modernos }\end{array}$ & $9(5.7 \%)$ & $37(23.6 \%)$ & $53(33.8 \%)$ & $\mathbf{3 9}(\mathbf{2 4 . 8 \% )}$ & $\mathbf{1 9}(\mathbf{1 2 . 1 \%})$
\end{tabular}




\begin{tabular}{|c|c|c|c|c|c|c|}
\hline \multirow{6}{*}{$\begin{array}{c}\text { tangible } \\
\text { s }\end{array}$} & Instalaciones físicas modernas & $13(8.3 \%)$ & $37(23.6 \%)$ & $42(26.8 \%)$ & $43(27.4 \%)$ & $22(14.0 \%)$ \\
\hline & $\begin{array}{l}\text { Uniforme de los trabajadores } \\
\text { adecuado }\end{array}$ & $16(10.2 \%)$ & $41(26.1 \%)$ & $51(32.5 \%)$ & $20(12.7 \%)$ & $29(18.5 \%)$ \\
\hline & $\begin{array}{l}\text { Equipamiento tecnológico } \\
\text { suficiente }\end{array}$ & $9(5.7 \%)$ & $52(33.1 \%)$ & $49(31.2 \%)$ & $40(25.5 \%)$ & $7(4.5 \%)$ \\
\hline & $\begin{array}{l}\text { Equipos visualmente } \\
\text { atractivos }\end{array}$ & $13(8.3 \%)$ & $28(17.8 \%)$ & $67(42.7 \%)$ & $26(16.6 \%)$ & $23(14.6 \%)$ \\
\hline & Limpieza y desinfección & $7(4.5 \%)$ & $74(47.1 \%)$ & $29(18.5 \%)$ & $25(15.9 \%)$ & $10(6.4 \%)$ \\
\hline & Instalaciones confiables & $15(9.6 \%)$ & $64(40.8 \%)$ & $40(25.5 \%)$ & $29(18.5 \%)$ & $9(5.7 \%)$ \\
\hline \multirow{4}{*}{$\begin{array}{l}\text { Fiabilid } \\
\text { ad }\end{array}$} & Confianza en el personal & $24(15.3 \%)$ & $47(29.9 \%)$ & $49(31.2 \%)$ & $20(12.7 \%)$ & $17(10.8 \%)$ \\
\hline & Información confiable & $15(9.6 \%)$ & $55(35.0 \%)$ & $50(31.8 \%)$ & $31(19.7 \%)$ & $6(3.8 \%)$ \\
\hline & Atención virtual & $17(10.8 \%)$ & $40(25.5 \%)$ & $43(27.4 \%)$ & $37(23.6 \%)$ & $20(12.7 \%)$ \\
\hline & Comunica pertinentemente & $21(13.4 \%)$ & $44(28.0 \%)$ & $42(26.8 \%)$ & $25(15.9 \%)$ & $25(15.9 \%)$ \\
\hline \multirow{6}{*}{$\begin{array}{l}\text { Capacid } \\
\text { ad de } \\
\text { respuest } \\
\text { a }\end{array}$} & Ofrece rapidez de respuesta & $20(12.7 \%)$ & $45(28.7 \%)$ & $47(29.9 \%)$ & $26(16.6 \%)$ & $21(13.4 \%)$ \\
\hline & Atención oportuna & $21(13.4 \%)$ & $46(29.3 \%)$ & $41(26.1 \%)$ & $29(18.5 \%)$ & $20(12.7 \%)$ \\
\hline & Disponibilidad de respuesta & $12(7.6 \%)$ & $56(35.7 \%)$ & $31(19.7 \%)$ & $38(24.2 \%)$ & $20(12.7 \%)$ \\
\hline & Resuelve reclamos & $17(10.8 \%)$ & $40(25.5 \%)$ & $35(22.3 \%)$ & $36(22.9 \%)$ & $29(18.5 \%)$ \\
\hline & Comportamiento & $17(10.8 \%)$ & $57(36.3 \%)$ & $51(32.5 \%)$ & $14(8.9 \%)$ & $16(10.2 \%)$ \\
\hline & Fiabilidad & $27(17.2 \%)$ & $39(24.8 \%)$ & $60(38.2 \%)$ & $15(9.6 \%)$ & $16(10.2 \%)$ \\
\hline \multirow{6}{*}{$\begin{array}{l}\text { Segurid } \\
\text { ad }\end{array}$} & Personal Capacitado & $25(15.9 \%)$ & $50(31.8 \%)$ & $48(30.6 \%)$ & $19(12.1 \%)$ & $15(9.6 \%)$ \\
\hline & $\begin{array}{l}\text { Actitud de orientación del } \\
\text { trabajador }\end{array}$ & $19(12.1 \%)$ & $42(26.8 \%)$ & $50(31.8 \%)$ & $32(20.4 \%)$ & $18(11.5 \%)$ \\
\hline & Comprensión del usuario & $9(5.7 \%)$ & $59(37.6 \%)$ & $29(18.5 \%)$ & $34(21.7 \%)$ & $26(16.6 \%)$ \\
\hline & Atención personalizada & $11(7.0 \%)$ & $54(34.4 \%)$ & $52(33.1 \%)$ & $20(12.7 \%)$ & $20(12.7 \%)$ \\
\hline & Horarios accesibles & $17(10.8 \%)$ & $58(36.9 \%)$ & $39(24.8 \%)$ & $27(17.2 \%)$ & $16(10.2 \%)$ \\
\hline & $\begin{array}{l}\text { Participación de las } \\
\text { festividades }\end{array}$ & $20(12.7 \%)$ & $41(26.1 \%)$ & $45(28.7 \%)$ & $33(21.0 \%)$ & $18(11.5 \%)$ \\
\hline \multirow[t]{5}{*}{ Empatía } & Seguimiento del servicio & $8(5.1 \%)$ & $38(24.2 \%)$ & $56(35.7 \%)$ & $32(20.4 \%)$ & $23(14.6 \%)$ \\
\hline & Modificación del servicio & $14(8.9 \%)$ & $47(29.9 \%)$ & $59(37.6 \%)$ & $21(13.4 \%)$ & $16(10.2 \%)$ \\
\hline & Protocolo de atención & $23(14.6 \%)$ & $39(24.8 \%)$ & $60(38.2 \%)$ & $18(11.5 \%)$ & $17(10.8 \%)$ \\
\hline & Simplificación de tramites & $23(14.6 \%)$ & $35(22.3 \%)$ & $49(31.2 \%)$ & $30(19.1 \%)$ & $20(12.7 \%)$ \\
\hline & Mejora de los procesos & $14(8.9 \%)$ & $63(40.1 \%)$ & $35(22.3 \%)$ & $23(14.6 \%)$ & $22(14.0 \%)$ \\
\hline
\end{tabular}

Fuente: Cuestionario a ciudadanos del distrito de Morales

\section{Interpretación:}

Respecto a la dimensión elementos tangibles, de los 157 ciudadanos el 56\% menciona estar "Algo y totalmente en desacuerdo" que los equipos tecnológicos son modernos, que las instalaciones físicas sean modernas (57\%) y que el equipamiento sea suficiente (44\%). Por otro lado, el 32.5\% indican que están "Ni en desacuerdo ni en acuerdo" con que el 
uniforme de los trabajadores sea adecuado y que los equipos tengan aspectos visualmente $\operatorname{atractivos}(43 \%)$.

Respecto a la dimensión fiabilidad, el 47\%, mencionan que están algo de acuerdo con la limpieza y desinfección de las instalaciones del municipio, el 41\%, indica que están algo de acuerdo en que las instalaciones son confiables, el 39.4\% también menciona los mismo, con que la información brindada por los trabajadores es confiable, el 31\%, indica que no están ni de acuerdo ni en desacuerdo con la confianza en el personal que atiende, finalmente el 36\% están "En desacuerdo y totalmente en desacuerdo" con la atención virtual que brinda el municipio.

Así también en la dimensión capacidad de respuesta, el 32\%, menciona que están "Algo desacuerdo y totalmente en desacuerdo" con la comunicación pertinente, que se ofrezca rapidez de respuesta (30\%), atención oportuna (31\%), disponibilidad de respuesta (37\%) y que el personal resuelva reclamos $(41 \%)$.

En cuanto a la dimensión Seguridad, el 36\% indican que se encuentran "Algo de acuerdo" con el comportamiento de los trabajadores respecto a los servicios brindados, fiabilidad en los trabajadores (25\%), personal capacitado (32\%); asimismo, mencionan que no se encuentran "Ni de acuerdo ni en desacuerdo" en cuanto a la actitud de orientación que tiene el trabajador respecto a los servicios brindados (32\%), están “Algo y Totalmente en desacuerdo" en cuanto a la comprensión de los usuarios (38\%).

Finalmente la dimensión Empatía, el 34.4\%, se encuentra "Algo de acuerdo" con la atención personalizada y con los horarios accesibles (36,4\%); el 33\% indican que están "Algo y totalmente en desacuerdo" en la participación de las festividades y fechas importantes del distrito compartidos por la institución, el 36\% mencionan estar "Ni de acuerdo ni en desacuerdo" en cuanto al seguimiento del servicio, a la modificación del servicio cuando el ciudadano lo solicita y en cuanto al cumplimiento del protocolo de atención por parte de los trabajadores del municipio (38\%) y simplificación de los tramites (32\%). Finalmente el 40\%, mencionan que están “Algo de acuerdo” con la mejora de los procesos de la Municipalidad.

Identificar los nudos críticos de gestión que dificultan la calidad de los servicios en el Municipio del Distrito de Morales. 
Figura 1. Nudos críticos que dificultan la calidad de servicio

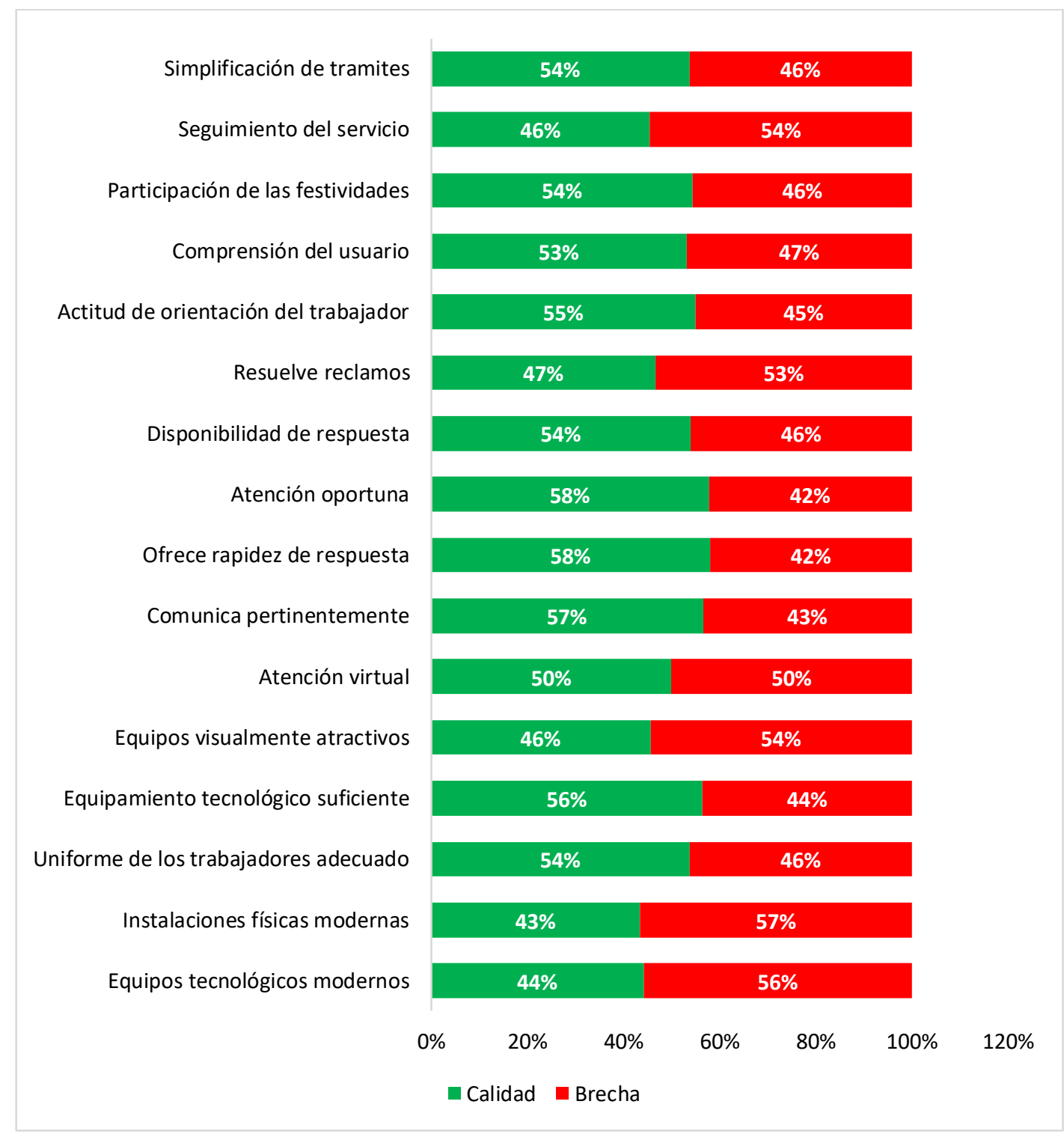

Fuente: Encuesta realizado al ciudadano del distrito de Morales

\section{Interpretación:}

Los nudos críticos que dificultan la calidad de los servicios en la dimensión de la empatía se encuentran identificadas, el $46 \%$ indica que existe deficiencias en la simplificación de trámites, deficiencia en el seguimiento de los servicios brindados (54\%), en la participación de las festividades del distrito (46\%), en la compresión del usuario (47\%) y en la orientación que brinda el trabajador a la hora de brindar el servicio (45\%).

En la dimensión seguridad, el $47 \%$ menciona que la compresión al usuario es un nudo crítico. 
De la misma manera en la dimensión capacidad de respuesta el 53\% encuentra deficiente a los trabajadores en cuanto a que resuelve reclamos por parte de los trabajadores, el $46 \%$ tiene disponibilidad de respuesta, el $43 \%$ se comunica pertinentemente.

Del mismo modo, los nudos críticos identificados en la dimensión fiabilidad, el 50\% tiene dificultades para acceder a los servicios a través de la atención virtual.

Finalmente, en la dimensión elementos tangibles el 54\% considera que los equipo no son visualmente atractivos, que se cuenta con equipamiento tecnológico suficiente (44\%), que los trabajadores no cuentan con uniforme adecuado (46\%), que las instalaciones y equipos tecnológicos no son modernas ( $57 \%$ y $56 \%$ ), respectivamente.

\section{Discusión}

En cuanto a la evaluación de la calidad de servicios se encontró que desde la percepcion de los ciudadanos son bajos y estos afectan el buen desarrollo de la institución. Según Mirabal et al., (2018), la realización del diagnóstico en el municipio los Palacios permitió constatar que predomina una visión sectorial de la calidad de vida por lo que se adolece de un proceso de gestión pública de la calidad de vida con enfoque integral desde los gobiernos locales y la Administración Pública. Igualmente Pastor, (2015), concluye que, los gobiernos locales se deben integrar a las necesidades de la población de tal manera que permita la elaboración de servicios acordes con los cambios y procesos de crecimiento de su entorno. Cuestiones que podemos entender que le falta realizar a los gobiernos locales con la finalidad de mejorar la calidad de servicios.

En cuanto a los nudos críticos encontrados luego de la evaluación, el $46 \%$ indica que existe deficiencias en la simplificación de trámites, el 54\% menciona sobre la deficiencia en el seguimiento de los servicios brindados, el $46 \%$ sobre las deficiencias en cuanto a la participación de las festividades del distrito, el $47 \%$ en cuanto a la compresión del usuario, el $45 \%$ en cuanto a la orientación que brinda el trabajador a la hora de brindar el servicio. En la dimensión seguridad, el 47\% menciona que la compresión al usuario es un nudo crítico. De la misma manera en la dimensión capacidad de respuesta en la identificación de los nudos críticos, el 53\% encuentra deficiente a los trabajadores en cuanto a que resuelve reclamos por parte de los trabajadores, el $46 \%$ tiene disponibilidad de respuesta, el $43 \%$ se comunica pertinentemente. Del mismo modo, los nudos críticos identificados en la dimensión fiabilidad, el 50\% tiene dificultades para acceder a los servicios a través 
de la atención virtual. Finalmente, en la dimensión elementos tangibles también se encontró con mayor incidencia nudos críticos, el 54\% considera que los equipo no son visualmente atractivos, el $44 \%$ considera que no se cuenta con equipamiento tecnológico suficiente, el $46 \%$ indica que los trabajadores no cuentan con uniforme adecuado, el $57 \%$ considera que las instalaciones no son modernas, el 56\% indica que los equipos tecnológicos que se utilizan para brindar los servicio no son modernos. Según Octavio \& Cruz, (2016), concluye que, el colaborador de la municipalidad que tiene altos conocimientos, buena actitud y aptitud, genera condiciones y aporta a mejorar la calidad de los servicios públicos por ello la importancia de fortalecer las competencias de los servidores municipales. Más del 50\% de las dimensiones e indicadores evaluados son relacionados a la capacidad que tenga el personal de la entidad a través de sus competencias y aptitudes para el logro de los resultados.

\section{CONCLUSIÓN O CONSIDERACIONES FINALES}

Se logró caracterizar la calidad del servicio municipal desde la perspectiva del ciudadano, en el distrito de Morales, a través del Modelo SERVQUAL, teniendo que en promedio los elementos tangibles el $42.7 \%$ se encuentran AED y TED; en fiabilidad el $53.1 \%$, capacidad de respuesta el $45.5 \%$, seguridad el $52.7 \%$ y empatía el $48.1 \%$ mencionan que están TDA y ADA.

Fueron identificados los nudos críticos de gestión que dificultan la calidad de los servicios en el Municipio del Distrito de Morales, en la dimensión empatía se encontraron 04 indicadores, siendo las más críticas con el 54\% el seguimiento del servicio. En la dimensión seguridad, solo se encontró 1 indicador, el 47\% menciona que la compresión al usuario es un nudo crítico; En cuanto a la capacidad de respuesta se identificó 3 nudos críticos, el 53\% indico las dificultades en resolver reclamos, mientras que en la dimensión fiabilidad se identificó con el $50 \%$ a la atención virtual como nudo crítico, en el caso de la dimensión elementos tangibles se encontró 05 nudos críticos, el 57\% se consideró que las instalaciones no son modernas.

Por lo cual se recomienda realizar una propuesta innovadora en la gestión de la calidad del servicio municipal del distrito de Morales, tomando en cuenta los nudos críticos identificados en la presente investigación que pueda dar solución a esta problemática. 


\section{LISTA DE REFERENCIAS}

Alam, M. S., \& Mondal, M. (2019). Assessment of sanitation service quality in urban slums of Khulna city based on SERVQUAL and AHP model: A case study of railway slum, Khulna, Bangladesh. Journal of Urban Management, 8(1), 20-27. https://doi.org/10.1016/j.jum.2018.08.002

Asubonteng, P., McCleary, K. J., \& Swan, J. E. (1996). SERVQUAL revisited: a critical review of service quality. Journal of Services Marketing, 10(6), 62-81. https://doi.org/10.1108/08876049610148602

Benito, B., Faura, Ú., Guillamón, M.-D., \& Ríos, A.-M. (2019). The efficiency of public services in small municipalities: The case of drinking water supply. Cities, 93, 95103. https://doi.org/10.1016/j.cities.2019.04.016

Bostanc1, B., \& Erdem, N. (2020). Investigating the satisfaction of citizens in municipality services using fuzzy modelling. Socio-Economic Planning Sciences, 69, 100754. https://doi.org/10.1016/j.seps.2019.100754

Dris Kharroub, A. O., \& COthmanMansour, M. M. (2019). The Impact of Strategic Planning in Palestinian Municipalities on the Quality of Service Provided to Its $\begin{array}{lllll}\text { Citizens. International Business } & \text { Research, }\end{array}$ https://doi.org/10.5539/ibr.v12n5p69

Durand Guevara, A. (2014). Movimientos sociales y política en el Perú de hoy. Latinoamérica. Revista de Estudios Latinoamericanos, 58, 59-84. https://doi.org/10.1016/s1665-8574(14)70101-2

Emrah, K., Y1ldırım, A., \& Ozcan, S. (2019). Integrated Usage of the SERVQUAL and Quality Function Deployment Techniques in the Assessment of Public Service Quality: The Case of Ardahan Municipality. Business and Economics Research Journal, 10(4), 885-901.

Eriksson, M., Persson Osowski, C., Malefors, C., Björkman, J., \& Eriksson, E. (2017). Quantification of food waste in public catering services - A case study from a Swedish municipality. Waste Management, 61, 415-422. https://doi.org/10.1016/j.wasman.2017.01.035 
Gobena, A. G. (2019). The Impact of Service Quality on Customer Satisfaction: A Case Study on Nekemte Municipality, Oromia Region, Ethiopia. Annals of Social Sciences \& Management studies, https://doi.org/10.19080/ASM.2019.04.555629

Idrovo-Toala, R. R., Allauca-Amaguaya, M., \& Chang-Camacho, L. B. (2020). Ciencias económicas y empresariales Artículo de investigación. Polo del Conocimiento, 5(10), 118-133. https://doi.org/10.23857/pc.v5i10.1792

INEI. (2018). Perú Indicadores de Gestión Municipal 2018.

Kansara, S. (2020). Modelling the water supply service quality: a case study of the municipal corporation. International Journal of Productivity and Quality Management, 29(1), 94. https://doi.org/10.1504/IJPQM.2020.104525

lo Storto, C. (2016). The trade-off between cost efficiency and public service quality: A non-parametric frontier analysis of Italian major municipalities. Cities, 51, 52-63. https://doi.org/10.1016/j.cities.2015.11.028

Mauro, S. G., Cinquini, L., \& Pianezzi, D. (2019). New Public Management between reality and illusion: Analysing the validity of performance-based budgeting. British Accounting Review, 100825. https://doi.org/10.1016/j.bar.2019.02.007

Mirabal, Y., Torres, C. C., \& Iglesias, A. (2018). Model for the public management from the quality of life to municipal scale in Cuba. Avances, 20(1), 40-51. https://dialnet.unirioja.es/servlet/articulo?codigo $=6513193$

Mhlongo, B. B., Nzimakwe, T. I., Naidoo, V., \& Munapo, E. (2016). Consumer Perceptions on Service Quality at Ethekwini Municipality within the Customer Service Centres. Journal of Social Sciences, 47(1), 79-89. https://doi.org/10.1080/09718923.2016.11893546

Moletsane, A. M., de Klerk, N., \& Bevan-Dye, A. L. (2014). Community expectations and perceptions of municipal service delivery: A case study in a South African municipality. Mediterranean Journal of Social Sciences, 5(21), 281-290. https://doi.org/10.5901/mjss.2014.v5n21p281 
Octavio, M., \& Cruz, T. (2016). The intellectual capital elemental factor for the quality of services and satisfaction of users of the provincial municipality of Puno. 1. http://www.scielo.org.pe/pdf/comunica/v7n1/a01v7n1.pdf

Parasuraman, A., Zeithaml, V. A., \& Berry, L. L. (1985). A Conceptual Model of Service Quality and Its Implications for Future Research. Journal of Marketing, 49(4), 4150. https://doi.org/10.1177/002224298504900403

Pastor-Seller, E. (2015). Opportunities for participation in the policies of municipal social services in Spain. Convergencia, 22(68), 229-257. http://www.scielo.org.mx/scielo.php?script=sci_arttext\&pid=S140514352015000200229

Psomas, E., Vouzas, F., Bouranta, N., \& Tasiou, M. (2017). Effects of total quality management in local authorities. International Journal of Quality and Service Sciences, 9(1), 41-66. https://doi.org/10.1108/IJQSS-04-2016-0035

Zhao, Y., Liu, L., Qi, Y., Lou, F., Zhang, J., \& Ma, W. (2020). Evaluation and design of public health information management system for primary health care units based on medical and health information. Journal of Infection and Public Health, 13(4), 491-496. https://doi.org/10.1016/j.jiph.2019.11.004

Živković, N., Glogovac, M., \& Kovačević, B. (2019). Determining the quality of municipal services using SERVQUAL model. Proceedings of the 5th IPMA SENET Project Management Conference (SENET 2019). https://doi.org/10.2991/senet19.2019 .4 